\title{
Projeto e construção de unidade de leitura de sensor Pirani para sistema de vácuo
}

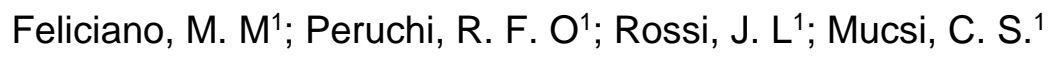 \\ 1 Instituto de Pesquisas Energéticas e Nucleares - IPEN - CNEN/SP
}

*e-mail: csmucsi@gmail.com

\begin{abstract}
Resumo
O presente trabalho desenvolveu um leitor de sensor Pirani com a utilização de um microcontrolador Atmel AVR de 8 bits. O microcontrolador utilizou o padrão de programação estruturada em linguagem $\mathrm{C}$, permitindo o uso de estruturas lógicas e cálculos aritméticos. Este comportamento foi utilizado para converter o sinal analógico proveniente do sensor, traduzindo-o para a apresentação em um painel de cristal líquido. O funcionamento de um sensor Pirani baseia-se na variação da resistência elétrica de uma dos ramos de uma ponte de Wheatstone em função da pressão gasosa. A tensão elétrica na ponte de Wheatstone foi mapeada para um valor de pressão gasosa, através da curva de calibração do sensor Pirani comercial, implementada no software da unidade de leitura. O equipamento desenvolvido apresentou uma performance adequada às demandas qualitativas da operação com custo de construção bastante atrativo.
\end{abstract}

\begin{abstract}
The Pirani vacuum gauges are used to measure pressure related to the density of gas molecules within a vessel, therefore this sensor is widely used in industrial process and equipment using vacuum. The study presents the development of a reading unit for a Pirani sensor controller using an Atmel AVR 8bit microcontroller. The $\mathrm{C}$ programming language is used allowing the processing of logic and arithmetic structures and converting the analog signal from the sensor. The results are displayed on an LCD panel. The Pirani gage works based on the extraction of heat by thermal conduction between a tungsten filament at high temperature and gases present in the vacuum system. The sensor has a Wheatstone bridge output voltage generated and mapped to the corresponding pressure value following the performance curve of the Pirani sensor implemented in software. The equipment presented adequate performance for the qualitative demands of the operation, with very attractive building cost.
\end{abstract}

Keywords (Palavras chaves): medidores de vácuo, micro controladores, Pirani.

\section{Introdução}

Os medidores de vácuo são amplamente utilizados em indústrias de metalurgia e em processos produtivos que possuam sistemas de vácuo. Os medidores Pirani têm o seu funcionamento baseado na variação de temperatura de um filamento de tungstênio, em função da quantidade de partículas do ambiente, de modo que as partículas colidam com o filamento e removam o calor. O filamento aquecido, do sensor
Pirani comercial, é conectado a uma ponte de Wheatstone e se comporta como um resistor não linear, não sendo expressa pela lei de Ohm; a sua resistência varia a partir de um coeficiente de temperatura do material e da variação de temperatura, causando um desequilíbrio na ponte e modificação da tensão de saída amplificada a partir de um circuito eletrônico interno.

\section{Materiais e Métodos}


O sensor Pirani Edwards APG100 NW16, mostrado na Figura 1, possui propriedades em estrutura proporcionando melhor funcionamento em relação à leitura, dois sensores de temperatura compensam a saída de modo que as mudanças de temperatura do ambiente de aplicação não interfiram significativamente no valor de leitura, três potenciômetros permitem ajustes de vácuo, pressão atmosférica e set-point do sensor fazendo com que a saída seja acionada quando o valor de pressão é menor do que o ponto de ajuste.

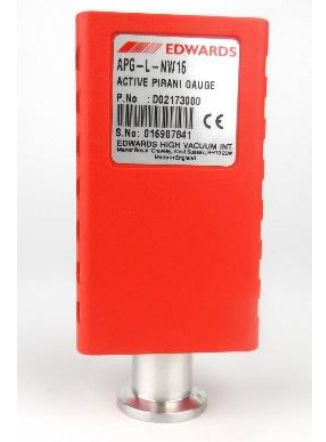

Figura 1. Sensor tipo Pirani modelo APG100 NW16, utilizado neste trabalho

O avanço da eletrônica e o fácil acesso à tecnologia permitiu o uso de sistemas micro controlados sem a necessidade de uma grande quantidade de componentes para realizar funções. Esta peculiaridade possibilita, ao micro controlador, o controle de entradas digitais e analógicas em que, através da programação, são utilizadas instruções para acionamento de saídas. Existem diversas famílias de microcontroladores disponíveis no mercado, como PIC, 8051 e ARM, entretanto em 2005 foi lançada a placa Arduino que atualmente é composta por um micro controlador da Atmel ATMega328, utilizando um conversor serial chipset FTDI ou um outro microcontrolador ATmega8U2 para comutação USB.

O sensor Pirani Edwards APG100 possui uma curva de performance apresentada em escala mono$\log$, Figura 2, que indica a relação entre o valor de tensão fornecido na saída e a pressão existente no local de medição. Esta curva é dividida em três trechos, sendo que à cada trecho é ajustada uma curva analítica, diminuindo assim o risco de acúmulo de erros. Estas curvas ajustadas são instaladas no software do micro controlador; à cada leitura de tensão realizada é, então associada uma pressão e apresentada no painel de cristal líquido.

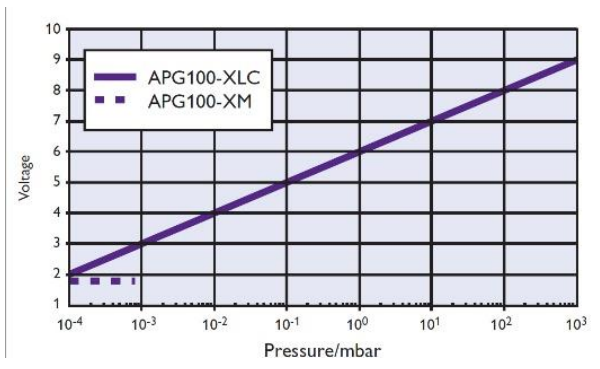

Figura 2. Curva de performance do sensor tipo Pirani modelo APG100 NW16, utilizado neste trabalho

Na Figura 3 é apresentado o circuito elétrico interligando o microcontrolador, e a entrada do sinal gerada pelo sensor.

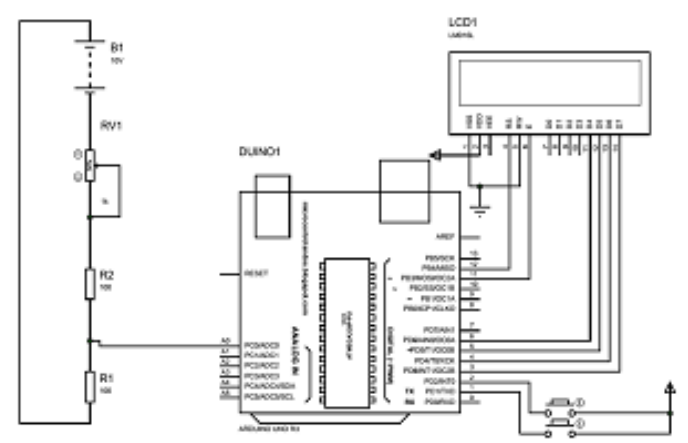

Figura 3. Circuito elétrico base de um leitor de um sensor Pirani.

O desenvolvimento de uma unidade microcontrolada capaz de realizar a leitura de um sensor Pirani Edwards AVG100, com a utilização do microcontrolador de baixo custo Atmel ATMega328 de 8 bits com clock de $16 \mathrm{MHz}$ permite que os valores de tensão sejam convertidos em pressão e posteriormente apresentados em um display de cristal líquido para controle de processo. O padrão de saída amplificada do sensor Pirani em tensão elétrica varia de $2 \mathrm{~V}$ a $10 \mathrm{~V}$ entretanto, a entrada analógica da placa Arduino permite uma tensão máxima de 5V. Faz-se, então, necessário, um divisor de tensão composto por resistores de precisão de $100 \Omega$ cada, para adequar a tensão e saída do sensor à tensão de entrada do microcontrolador, na Figura 4 ilustra-se a montagem em fase inicial em uma protoboard utilizando uma fonte com tensão variável com comportamento semelhante ao sensor. 


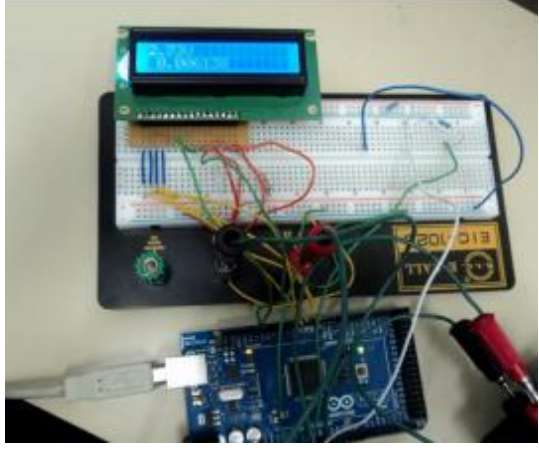

Figura 4. Montagem do circuito divisor de tensão e o painel de cristal líquido na protoboard.

\section{Resultados e Discussões}

Os microcontroladores em geral podem ser programados com linguagens de baixo nível que exigem menor tempo de processamento, como a linguagem Assembler, um nível acima da linguagem de máquina, entretanto a linguagem de alto nível permite maior similaridade à linguagem humana, possibilitando a programação estruturada, mais utilizada para microcontroladores. A programação do Arduino é realizada através de um ambiente integrado de desenvolvimento (IDE), baseada nas linguagens $C$ e $\mathrm{C}++$, permitindo instruções lógicas e cálculos aritméticos.

O divisor de tensão pôde ser montado em uma placa de fenolite cobreada perfurada, junto ao ajuste da retroiluminação do painel de cristal líquido e sua conexão ao microcontrolador, conforme apresentado na Figura 5.

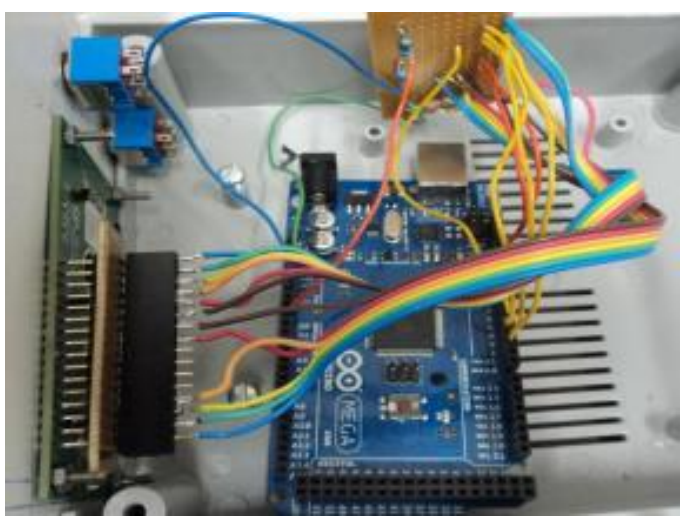

Figura 5. Montagem em fase final do leitor

\section{Conclusões}

Atualmente as placas Arduino não são utilizadas na indústria por ser pouco robusto e trabalhar com tensões baixas, porém atendem adequadamente no desenvolvimento de projetos. O micro controlador AVR Atmel utilizado no desenvolvimento do leitor para o sensor Pirani apresentou desempenho adequado às demandas qualitativas da operação com diferenças significativas de custos se comparado a um controlador industrial. A placa possui grande flexibilidade de acesso às funções, operação e programação sem a necessidade do desenvolvimento de bibliotecas específicas, possibilitando o aperfeiçoamento com novas aplicações a partir da aquisição de módulos de funcionalidades, como o Ethernet Shield que possibilita comunicação Wireless com demais dispositivos e o Módulo Relé que permite o acionamento de saídas digitais. A leitura do sensor Pirani pode variar de acordo com a taxa de transferência de calor dos gases, o microcontrolador pode ser reprogramado através da conexão USB, sem que seja necessário um gravador de microcontrolador adicional, permitindo a adequação do leitor para diferentes atmosferas, que alteram sensivelmente seu desempenho, ilustra-se na Figura 6 este comportamento.

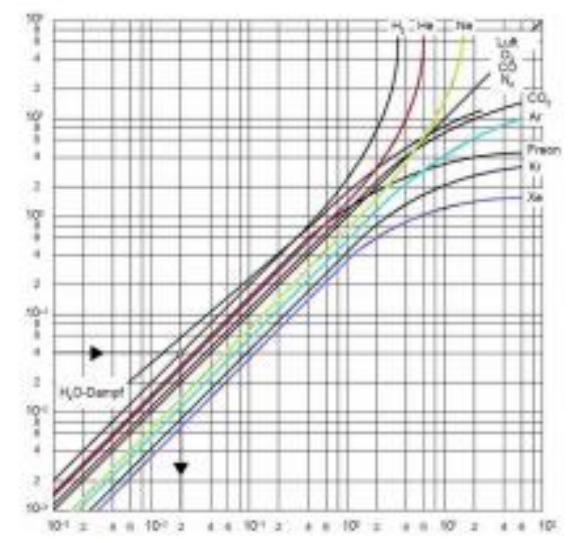

Figura 6. Curvas de conversão para a leitura realizada num ambiente diferente do Nitrogênio.

\section{Referências}

[1] KIENITZ, K. H; Analise de circuitos um enfoque em sistemas. São Paulo: Editora Malone Ltda: 2002.

[2] UNIVERSIDADE DE SÃO PAULO. Instituto de física. Medidores de vácuo, São Paulo. 1992.

[3] Visão geral sobre a tecnologia do vácuo, São Paulo. Disponível em:

<http://www.sensum.net/public_html/materia01.html>. Acesso em: 05 Mar.2016.

[4] https://www.avtreliability.com/wpcontent/uploads/APG100-series-vacuum-gaugesdatasheet.pdf - Acesso em 1 de Nov. de 2016. 